\title{
Maintenance of undifferentiated state of human induced pluripotent stem cells through cytoskeleton-driven force acting to secreted fibronectin on a dendrimer-immobilized surface
}

\author{
Mee-Hae Kim and Masahiro Kino-oka* \\ Department of Biotechnology, Graduate School of Engineering, Osaka University, 2-1 Yamadaoka, \\ Suita, Osaka 565-0871, Japan
}

*Correspondence: Masahiro Kino-oka, Ph.D.

Tel.: +81-(0)6-6879-7444

Fax: +81-(0)6-6879-4246

E-mail: kino-oka@bio.eng.osaka-u.ac.jp

Running title: Maintatenace of hiPSCs on dendrimer surface 


\section{ABSTRACT}

Understanding of the fundamental mechanisms that govern adhesive properties of human induced

pluripotent stem cells (hiPSCs) to culture environments provides surface design strategies for maintaining their undifferentiated state during cell expansion. Polyamidoamine dendrimer surface with first-generation (G1) with dendron structure was used for co-cultures of hiPSCs and SNL feeder cells that formed tightly packed compact hiPSC colonies, similar to those on a conventional gelatin-coated surface. hiPSCs passaged up to 10 times on the G1 surface maintained their undifferentiated state. Immunostaining and RT-PCR analysis of fibronectin showed that the secreted fibronectin matrix from feeder cells on the G1 surface contributed to hiPSC attachment. Compared with cells on the gelatin-coated surface, F-actin and paxillin immunostaining revealed a well-organized network of actin stress fibers and focal adhesion formation at cell-substrate sites in hiPSC colonies on the G1 surface. E-cadherin expression levels on these surfaces were almost same, but paxillin and Rac1 expression levels on the G1 surface were significantly higher than those on the gelatin-coated surface. Zyxin showed prominent expression on the G1 surface at sites of focal adhesion and cell-cell contact in colonies, whereas zyxin expression on the gelatin-coated surface was not observed in regions of cell-cell contact. These findings indicate that transduction of mechanical stimuli through actin polymerization at sites of focal adhesion and cell-cell contact results in maintenance of undifferentiated hiPSC colonies on G1 surface. The G1 surface enables a substrate design based on the mechanical cues in the microenvironment from feeder cells to expand undifferentiated hiPSCs in long-term culture.

[Keywords: Human induced pluripotent stem cells; undifferentiation; mechanotransduction; cell-cell interaction; dendrimer surface] 


\section{INTRODUCTION}

Human induced pluripotent stem cells (hiPSCs) are genetically reprogrammed to an embryonic stem cell-like state by forced gene expression of factors that are important to maintain the defining properties of human embryonic stem cells (1-3). hiPSCs are characterized by their capacity to differentiate into cell types of all three germ layers (3). Because of their self-renewing and pluripotency, hiPSCs are a promising resource for many areas of research and medicine $(1,2)$.

Expansion of hiPSCs in an undifferentiated state remains challenging because spontaneous differentiation occurs during serial subcultures, resulting in the loss of their self-renewal and pluripotent qualities. Therefore, vigilant efforts to maintain the undifferentiated state are one of the major requirements. hiPSC culture requires the support of mouse or human fibroblasts for attachment and maintenance of pluripotency $(4,5)$. It is thought that feeder cells secrete various growth factors and extracellular matrices to support cell growth and maintenance of the undifferentiated state. Because signaling molecule responses are affected by interactions between the cells and the surrounding matrix, many studies have investigated the effects of the extracellular matrix secreted from feeder cells on the maintenance of undifferentiated hiPSCs during long-term culture (6). Recent reports have focused on the opportunity to tune the properties of synthetic substrates to better mimic the microenvironments of feeder cells for the long-term maintenance of undifferentiated hiPSCs in vitro (7-10). In this context, hiPSC culture has been advanced significantly by identification of essential growth factors and specific substrate coatings that allow for cellular growth, survival, and maintenance of the undifferentiated state $(6,8)$.

For the design of a synthetic culture surface to culture undifferentiated hiPSCs, many studies have been developed stem cell culture to mimic cell-cell and cell-matrix interactions and modulate stem cell self-renewal and differentiation characteristic of stem cell microenvironments (8-10). The strategies include influencing the cell-cell interactions and cellular interactions with the culture substrate through the physical properties of substrates, including topography, roughness, and elasticity (8). The transmission of mechanical forces from outside the cell through cell-substrate and 
1 cell-cell interactions appears to control the maturation or disassembly of these adhesions and

2 initiates intracellular signaling cascades that ultimately alter stem cell fate $(6,9)$. These studies have

3 observed how stem cells respond to these cues and started to shed light on the pathways that dictate

4 stem cell survival, self-renewal, and lineage commitment. The mechanisms by which mechanical

5 cues from neighboring cells and the substrate activates the intracellular signaling pathways are

6 widely studied in many cell types, but there have been few studies in hiPSCs.

7 In previous studies, we have investigated to design synthetic culture surfaces using a

8 dendrimer substrate to create suitable microenvironments that regulate morphology and cellular

9 function (11-14). Polyamidoamine dendrimer surfaces with dendron structure have been used to

10 regulate the morphology and function of stem cells (11). In this study, we examined the

11 morphological and functional responses of hiPSCs cultured on the dendrimer-immobilized surface.

12 Moreover, fundamental mechanisms of cell and surface interaction are discussed in terms of 13 cytoskeletal organization and focal adhesion formation to pluripotency maintenance of hiPSCs. The 14 obtained results lead us to propose a synthetic culture surface to maintain the undifferentiated state 15 of hiPSCs in long-term culture.

\section{MATERIALS AND METHODS}

19 culture of were conducted in $0.1 \%$ gelatin-coated culture dish (surface area; $55-\mathrm{cm}^{2}$, Corning Costar, 20 Cambridge, MA, USA) on a feeder layer of mitomycin C-treated SNL76/7 cells (European 21 Collection of Cell Cultures, Salisbury, UK) using commercial medium (ReproStem, ReproCELL 22 Inc., Tokyo) containing $5 \mathrm{ng} / \mathrm{ml}$ basic fibroblast growth factor. Cells were cultured at $37^{\circ} \mathrm{C}$ with $5 \%$ $23 \mathrm{CO}_{2}$ atmosphere as described previously (15). Culture medium was replenished every day.

24 To subculture hiPSCs, the cells were washed once with phosphate-buffered saline (PBS, 25 Sigma-Aldrich, MO, USA) and then incubated with CTK solution as described elsewhere (2). 
1 hiPSC colonies in undifferentiated state were collected carefully by using cell scraper (Sumitomo

2 Bakelite Co., Ltd. Osaka). The suspension of collected undifferentiated colonies was pipetted gently

3 into small aggregates, and the suspension was dispersed into fresh culture vessel containing feeder

4 cells.

5 To prepare feeder layers, mitomycin C-treated SNL76/7 cells were cultured for $24 \mathrm{~h}$ in

6 Dulbecco's modified Eagle's medium (DMEM, Sigma-Aldrich) supplemented with 7\% fetal bovine

7 serum (FBS, Invitrogen, Grand Island, NY, USA) with antibiotics (100 U/ $\mathrm{cm}^{3}$ penicillin G, 0.1

$8 \mathrm{mg} / \mathrm{cm}^{3}$ streptomycin and $0.25 \mathrm{mg} / \mathrm{cm}^{3}$ amphotericin B; all obtained from Invitrogen). Seeding

9 density was fixed at a viable cell concentration of $2.5 \times 10^{4}$ cells $/ \mathrm{cm}^{2}$.

10 Cell culture on dendrimer surface The first-generation dendrimer (G1) surface was

11 prepared using the conventional tissue culture polystyrene (PS) surface of a square 8-well plate

12 (surface area; $10.5 \mathrm{~cm}^{2}$, Nunc, Roskilde, Denmark), as described previous report (10). Briefly,

13 hydroxyl groups were displayed on the PS surface by pouring potassium tert-butoxide into the wells.

14 Then, aqueous glutaraldehyde was introduced into the wells, with the wells were then treated with a

15 tris(2-aminoethyl) amine solution to produce a dendron structure. The wells were rinsed with sterile

16 water. To display glucose as a terminal ligand, D-glucose was applied.

17 For all experiments, at 1 day before plating hiPSCs, the SNL feeder cells were cultured on the

18 G1 surface, and then hiPSCs were plated on the conditioned G1 surface with feeder cells under

19 same conditions as those described above. Colonies deviated from undifferentiated state were not

20 removed before passaging the cells during serial passages.

21 Immunofluorescence staining The procedure used for immunofluorescence staining

22 was similar to a previously described method (12). Briefly, hiPSCs were fixed with $3.7 \%$

23 paraformaldehyde (Wako Pure Chemical Industries, Osaka) for $10 \mathrm{~min}$ at room temperature and

24 rinsed with PBS, followed by soaking in PBS with $0.25 \%$ Triton X-100 for 4 min. After masking of

25 non-specific proteins by incubation in Block Ace (Dainippon Sumitomo Pharma Co., Ltd., Osaka)

26 for $1 \mathrm{~h}$ at ambient temperature, the cells were treated with a primary antibody at $4{ }^{\circ} \mathrm{C}$ overnight. 
1 The cells were incubated with anti-Oct3/4 (Santa Cruz Biotechnology, CA, USA), anti-Nanog

2 (Stemgent, San Diego, CA, USA), anti-SSEA-4 (Stemgent), anti-Tral-60 (Stemgent),

3 anti-fibronectin (Santa Cruz Biotechnology), anti-paxillin (Millipore), and anti-zyxin (Abcam,

4 Cambridge, MA, UK) primary antibodies that were adequately diluted in PBS containing $10 \%$

5 Block Ace. Cells were washed with Tris-buffered saline followed by immunolabeling with Alexa

6 Fluor 488-conjugated goat anti-rabbit or Alexa Fluor 594-conjugated goat anti-mouse IgG

7 (Molecular Probes, Eugene, OR, USA) for $1 \mathrm{~h}$. F-actin and cell nuclei were stained with rhodamine

8 phalloidin (Molecular Probes) and DAPI (4',6-diamidino-2-phenylindole), respectively. Images

9 were obtained using an image analyzer (In CELL Analyzer 2000; GE Healthcare, Buckinghamshire,

10 UK) through $10 \times$ objective lens and confocal laser scanning microscope (Model FV-1000; Olympus,

11 Tokyo) through $60 \times$ objective lens.

RNA isolation and reverse transcriptase PCR (RT-PCR) analysis RNA isolation,

14 isolated using an RNeasy Mini Kit (Qiagen, Hilden, Germany) and TRIzol (Invitrogen). One $\mu \mathrm{g}$ of 15 total RNA was then reverse transcribed using SuperScript II Reverse Transcriptase kit (Invitrogen).

16 The qPCR assays were conducted using SYBR Premix Ex Taq (Takara Shuzo, Shiga, Japan), and 17 contained $1 \mu 1$ of cDNA per $25 \mu 1$ of reaction mixture. Assays were carried out on a 7300 Real-Time 18 PCR System (Applied Biosystems, Foster City, CA, USA). The relative quantity of the target 19 transcript was estimated by a standard curve, and the data were standardized based on the GAPDH 20 expression. All PCR products were checked by melting curve analysis to exclude the possibility of 21 multiple products or incorrect product size. The primer sequences are listed in Supplementary table $22 \mathrm{~S} 1$.

RESULTS

24 Long-term maintenance of undifferentiated hiPSCs Morphologies of hiPSCs and their 25 colonies cultured on the G1 and gelatin-coated surfaces with feeder cells for 5 days were compared 
1 as indicated in Fig. S1. Growth on gelatin was used as a reference condition to compare the growth

2 and maintenance of hiPSCs on the G1 surface. Similar to undifferentiated hiPSCs cultured on the

3 gelatin-coated surface, hiPSCs on the G1 surface grew as tight colonies with cells densely packed

4 together. hiPSCs on the G1 surface maintained their characteristic morphology after 10 passages (50

5 days).

6 To confirm the phenotypes of hiPSCs cultured on the G1 and gelatin-coated surfaces in serial 7 passages for long-term culture, we performed immunostainings of pluripotency markers, Oct3/4, Nanog, SSEA-4, and Tra1-60 of hiPSCs after 10 passages. As shown in Fig. 1A, the colonies passaged on the G1 or gelatin-coated surfaces were strongly positive, and the majority of cells in the colonies were stained for these markers. expression levels of pluripotency and early differentiation markers, by RT-PCR analyses after 1 passage and 10 passages. The gene expression of pluripotency markers (Oct3/4, Nanog, Sox2) in surface. To verify the matrix organization of secreted fibronectin from feeder cells and their expression levels during hiPSC culture, cells cultured on the G1 and gelatin-coated surfaces with feeder cells for 5 days were analyzed by immunostainings and RT-PCR analysis. As shown in Fig. 2A, fibronectin secreted on 
1 gelatin-coated surface. Additionally, the comparison for the expression level of fibronectin did not

2 show significant difference in fibronectin mRNA levels (Fig. 2B).

4 assess the cytoskeletal organization accompanied by focal adhesion formation of hiPSCs, cells

5 cultured on the G1 and gelatin-coated surfaces with SNL feeder cells for 5 days were

6 immunostained for F-actin and paxillin, a focal adhesion protein. As shown in Fig. 3, the Fig. 3

7 circumferential actin bundles were well recognized in the apical side of hiPSC colonies, and the staining patterns were similar on the two surfaces. However, many stress fibers appeared in the basal side of central and peripheral regions in hiPSC colonies on the G1 surface, and some stress fibers stretching across the cytoplasm were developed, compared with those on the gelatin-coated surface. Moreover, paxillin was diffusedly distributed through the cytoplasm of cells in the basal side of colonies on the G1 surface, especially at the ends of stress fibers with intensive staining in the longitudinal direction in a scattered manner (Fig. 3D3). hiPSCs on the G1 surface showed increased paxillin staining of large adhesions, compared with those on the gelatin-coated surface. These observations indicate enhanced actin filament networks and focal adhesion formation of hiPSCs when they are grown on the G1 surface.

We also examined the expression levels of paxillin, Rac1, and E-cadherin in hiPSCs cultured on G1 and gelatin-coated surfaces with feeder cells for 5 days by western blot analysis. As shown in Fig. 4, the expression levels for paxillin and Rac1 in cells cultured on the G1 surface were Fig. 4 20 significantly higher than those in cells cultured on the gelatin-coated surface. Furthermore, 21 E-cadherin protein levels showed no significant difference in cells on the G1 and gelatin-coated surfaces.

To investigate the mechanical properties in hiPSCs, we examined the distribution of zyxin, a cytoskeletal protein, in hiPSCs cultured on the G1 and gelatin-coated surfaces with feeder cells. As shown in Fig. 5, zyxin expression on the G1 surface appeared to localize primarily at the sites of Fig. 5 focal adhesion and cell-cell contacts in both the central and peripheral regions of colonies. However, 
1 zyxin expression on the gelatin-coated surface was found only in the basal side with small scattered

2 spots of staining. By comparing G1 and gelatin-coated surfaces, we found that zyxin expression was

3 much stronger in cells cultured on the G1 surface.

\section{DISCUSSION}

\section{Adhesion of hiPSCs to the dendrimer surface is facilitated by secretion of fibronectin} matrix from feeder cells It has been well documented that the in vitro expansion of hiPSCs relies on cell-substrate interaction that occurs between integrin and extracellular adhesion protein. Maintenance of the undifferentiated state and self-renewal of hiPSCs requires a co-culture environment in monolayer expansion, which is achieved by culturing on a layer of feeder cells (5). The secreted growth factors and extracellular matrices from feeder cells serve dual roles to support the attachment and maintenance of undifferentiated hiPSCs. Once feeder cells are attached to the culture surface, they begin to secrete and remodel extracellular matrix proteins, such as laminin, fibronectin, and vitronectin, to which integrin bind. One protein of particular interest to determine the relationship between cells and their substratum is fibronectin, because it is one of the major molecules that mediate cell attachment (16). Fibronectin might be affected by substrata that alter the distribution of cytoskeletal elements. This activity may provide fibronectin with the ability to mediate cell-substrate and cell-cell interactions. The cell-substrate and cell-cell interactions activate specific mechanotransduction pathways that control cell survival, self-renewal, and lineage commitment $(17,18)$.

In our previous study, we found that cell and colony morphology of hiPSCs associated with altered migration on the dendrimer surfaces were responsible for the coordinated regulation of balance between cell-cell and cell-substrate interactions, thereby switching their transition from self-renewal state to early endoderm differentiation in hiPSCs (14). hiPSCs cultured on the G1 surface for 5 days were maintained in an undifferentiated state, while those on the G5 surface exhibited the early commitment to differentiation toward endodermal fates. In this study, the G1 
1 surface was used for co-cultures of hiPSCs and feeder cells during serial subcultures. hiPSCs on the

2 G1 surface were stably maintained for up to 10 passages without loss of their typical hiPSC

3 morphology, similar to those on a conventional gelatin-coated surface (Fig. 1). To determine the

4 effect of substratum surface topography on hiPSCs colony formation and changes in cell

5 morphology on the major extracellular adhesive proteins, we investigated the organization and

6 expression of fibronectin at protein and mRNA level. Fibronectin on G1 surface had a diffuse

7 distribution over the entire cells in the form of spots, similarity to those on gelatin-coated surface

8 (Fig. 2A). There are no significant differences in fibronectin mRNA levels (Fig. 2B). These findings

9 suggest that colony formation of hiPSCs on a G1 surface used as a synthetic substrate is regulated

10 by the actin cytoskeletal architecture and focal adhesion formation through feeder cells associated

11 with fibronectin.

Cytoskeleton-driven force acting through focal adhesion sustains the undifferentiated state of hiPSCs effectively during long-term culture Adhesion of stem cells to the 14 underlying culture surface modulates cell growth and differentiation through signaling 15 reorganization of the actin cytoskeleton $(17,18)$. The interaction between integrin and an intact 16 cytoskeleton as well as the assembly of actin stress fibers and cell-generated tension are all 17 thought to be important factors regulating cell fate decisions (19). Formation of the 18 integrin-mediated adhesions regulated focal adhesion-associated signaling molecules, paxillin and 19 zyxin, to coordinate spatiotemporal activation of a member of Rho family GTPases, Rac1 and their 20 associated effectors to regulate cell migration (19-21). Paxillin and zyxin have been suggested to 21 mediate an important subset of cellular responses to force during migration $(22,23)$. They localize 22 to the focal adhesions of adherent cells and localize to focal adhesion and cell-cell contacts to 23 regulate cytoskeletal organization and cell motility $(23,24)$. Transmission of forces from outside 24 the cell through cell-substrate and cell-cell contacts appears to control the maturation or 25 disassembly of these adhesions and initiates intracellular signaling cascades that ultimately alter 26 many cellular behaviors. In response to externally applied forces, cells actively rearrange the 
1 organization and contractile activity of the cytoskeleton and redistribute their intracellular forces

$2(23,25)$. The cytoskeleton has been shown to bind to factors that control gene transcription

3 (21-23). These studies have provided our current understanding of the focal adhesion mechanism

4 to culture surfaces that functions as an adaptor protein to recruit diverse cytoskeleton and signaling

5 proteins into a complex.

6 In this study, we showed that the secreted fibronectin matrix on the G1 surface was tightly

7 coupled to events that play an important role not only in signaling at cell-substrate adhesions but

8 also at the cell-cell contacts of hiPSCs (Fig. 2). In addition, we found that the cells cultured on the

9 G1 surface had many stress fibers and focal adhesions with paxillin compared with those of cells on

10 the gelatin-coated surface (Fig. 3). Western blot analyses of the expression levels of paxillin, Rac1,

11 and E-cadherin revealed an increase of paxillin and Rac1 in the cells cultured on the G1 surface, but

12 the E-cadherin protein level showed no significant difference in the cells on the G1 and

13 gelatin-coated surfaces (Fig. 4). Our results suggest that the G1 surface induces increased turnover

14 of focal adhesion to promote migration during hiPSC colony formation. Zyxin in cells on the G1

15 surface appeared at sites of cell-substrate and cell-cell contacts of hiPSCs in colonies on the G1

16 surface (Fig. 5C and 5D), whereas the zyxin on gelatin-coated surface could only be detected in

17 these location at cell-substrate adhesion (Figs. 5A and 5B). It is noteworthy that zyxin shuttles

18 between the sites of cell-substrate adhesion and cell-cell contacts of hiPSCs in colonies on the G1

19 surface. We propose that zyxin may regulate whether cell migration occurs independently in

20 individual cells or is coordinated inside colonies that maintain balance between cell-substrate and

21 cell-cell interactions. Zyxin is an alpha-actinin and stress-fiber-binding protein found in mature

22 focal contacts $(23,24)$. Furthermore, zyxin has been shown to bind to factors that control gene

23 transcription and they function as regulatory domains for controlling protein-protein interactions

24 with components involved in cell-cell interaction. Our results suggest that maintenance of

25 undifferentiated hiPSCs on the G1 surface is regulated by the well-organized actin cytoskeletal

26 architecture through focal adhesion formation, which in turn elicit differential contractile forces and 
1 adhesion to stimulate cell migration. An important consideration in hiPSC culture is that cell-cell

2 contractility within growing hiPSC colonies may change local region inside colonies, which may

3 affect maintenance of undifferentiated state. When the hiPSCs grown on the G1 and gelatin-coated

4 surfaces were compared, cells on the G1 surface are facilitated by mechanical forces acting through

5 zyxin with respect to attachments of the actin cytoskeleton to regions of cell-substrate and cell-cell

6 contacts. The effects of mechanical signaling through cytoskeleton in hiPSCs on the G1 surface

7 indicate that modulation of zyxin expression is sufficient to sustain their undifferentiated state. The

8 mechanisms by which mechanical cues from neighboring cells and the substrate facilitate the

9 spreading and self-renewal of hiPSCs are reported in other study. Jin et al. show that hiPSCs can be

10 maintained undifferentiated state on the synthetic peptide engineered surface (26). They showed

11 that the cytoskeleton characterization of hiPSCs grown on the synthetic peptide substrate revealed

12 formation of denser actin filaments and up-regulation of zyxin expression in the cell-cell contacts. It

13 is likely to interact with transcription factors to regulate gene expression associated with

14 maintenance of undifferentiated hiPSCs and its differentiation capacity $(18,27)$. While the

15 mechanism of these changes in cell cytoskeletal formation is unclear, it may still indicate a

16 reorganization of focal adhesions, which facilitates the spreading and self-renewal of hiPSCs on G1

17 surface.

18 In conclusion, the G1 surface can be used as an artificial substrate to support in vitro expansion

19 of hiPSCs while maintaining their undifferentiated state. hiPSCs cultured on the G1 surface formed

20 tight compact colonies that showed maintenance of undifferentiated characteristics similar to those

21 of cells cultured on gelatin-coated surface. We have shown that feeders-secreted fibronectin on G1

22 surface enhanced the availability of integrin-mediated adhesion of hiPSCs with enhancement of

23 actin polymerization at sites of focal adhesion and cell-cell contact. In addition, the cytoskeletal and

24 focal adhesion formation on this surface is responsible for the increased zyxin expression involved

25 in transducing mechanical stimuli, thereby facilitating maintenance of undifferentiated state of

26 hiPSCs in long-term culture. The applied culture surface may be a useful alternative to culture 
1 hiPSCs in an undifferentiated state.

\section{ACKNOWLEDGMENTS}

3 This work was partially supported by the Japan Society for the Promotion of Science (JSPS)

4 KAKENHI, Grants-in-Aid for Young Scientists (B) (Nos. 23760749 and 25820401) and

5 Grant-in-Aid for Challenging Exploratory Research (No.25630377). Our work was also supported

6 by the Strategic Promotion of Innovative Research and Development (S-Innovation) Program of the

7 Japan Science and Technology Agency (JST), and the JSPS through the "Funding Program for

8 World-Leading Innovative R\&D on Science and Technology (FIRST Program)" initiated by the

9 Council for Science and Technology Policy (CSTP). 
1. Takahashi, K. and Yamanaka, S.: Induction of pluripotent stem cells from mouse embryonic and adult fibroblast cultures by defined factors, Cell, 126, 663-676 (2006).

2. Takahashi, K., Tanabe, K., Ohnuki, M., Narita, M., Ichisaka, T., Tomoda, K., and Yamanaka, S.: Induction of pluripotent stem cells from adult human fibroblasts by defined factors, Cell, 131, 861-872 (2007).

3. Wernig, M., Meissner, A., Foreman, R., Brambrink, T., Ku, M., Hochedlinger, K., Bernstein, B.E., and Jaenisch, R.: In vitro reprogramming of fibroblasts into a pluripotent ES-cell-like state, Nature, 448, 318-324 (2007).

4. McKay, T.R., Camarasa, M.V., Iskender, B., Ye, J., Bates, N., Miller, D., Fitzsimmons, J.C., Foxler, D., Mee, M., Sharp, T.V., Aplin, J., Brison, D.R., and Kimber, S.J.: Human feeder cell line for derivation and culture of hESc/hiPSc, Stem Cell Res., 7(2):154-162 (2011).

5. Keshel, S.H., Soleimani, M., Tavirani, M.R., Ebrahimi, M., Raeisossadati, R., Yasaei, H., Afsharzadeh, D., Behroz, M.J., Atashi, A., Amanpour, S., Khoshzaban, A., Roozafzoon, R., and Behrouzi, G.R.: Evaluation of unrestricted somatic stem cells as a feeder layer to support undifferentiated embryonic stem cells, Mol. Reprod. Dev., 79, 709-718 (2012).

6. Fuchs, E., Tumbar, T., and Guasch, G.: Socializing with the neighbors: Stem cells and their niche, Cell, 116(6), 769-778 (2003).

7. Rodin, S., Domogatskaya, A., Ström, S., Hansson, E.M., Chien, K.R., Inzunza, J., Hovatta, O., and Tryggvason, K.: Long-term self-renewal of human pluripotent stem cells on human recombinant laminin-511, Nat. Biotechnol., 28, 611-615 (2010).

8. Metallo, C.M., Mohr, J.C., Detzel, C.J., de Pablo, J.J., Van Wie, B.J., and Palecek, S.P.: Engineering the stem cell microenvironment, Biotechnol. Prog., 23(1), 18-23 (2007).

9. Lutolf, M.P., Gilbert, P.M., and Blau, H.M.: Designing materials to direct stem-cell fate, Nature, 462, 433-441 (2009).

10. Marklein, R.A. and Burdick, J.A.: Controlling stem cell fate with material design. Adv. 
Mater., 22, 175-189 (2010).

11. Kim, M.H., Kino-oka, M., and Taya, M.: Designing culture surfaces based on cell anchoring mechanisms to regulate cell morphologies and functions, Biotechnol. Adv., 28, 7-16 (2010).

12. Kim, M.H., Kino-oka, M., Maruyama, N., Saito, A., Sawa, Y., and Taya, M.: Cardiomyogenic induction of human mesenchymal stem cells by altered Rho family GTPase expression on dendrimer-immobilized surface with D-glucose display, Biomaterials, 31, 7666-7677 (2010).

13. Mashayekhan, S., Kim, M.H., Miyazaki, S., Tashiro, F., Kino-oka, M., Taya, M., and Miyazaki, J.: Enrichment of undifferentiated mouse embryonic stem cells on a culture surface with a glucose-displaying dendrimer, Biomaterials, 29, 4236-4243 (2008).

14. Kim, M.H. and Kino-oka, M.: Switching between self-renewal and lineage commitment of human induced pluripotent stem cells via cell-substrate and cell-cell interactions on a dendrimer-immobilized surface, Biomaterials, 35, 5670-5678 (2014).

15. Kim, M.H., Masuda, E., and Kino-oka, M.: Kinetic analysis of deviation from the undifferentiated state in colonies of human induced pluripotent stem cells on feeder layers, Biotechnol. Bioeng., 11, 1128-1138 (2014).

16. Chou, L., Firth, J.D., Uitto, V.J., and Brunette, D.M.: Substratum surface topography alters cell shape and regulates fibronectin mRNA level, mRNA stability, secretion and assembly in human fibroblasts, J. Cell Sci., 108, 1563-1573 (1995).

17. Delon, I. and Brown, N.H.: Integrins and the actin cytoskeleton, Curr. Opin. Cell Biol., 19(1), 43-50 (2007).

18. Sepulveda, J.L., Gkretsi, V., and Wu, C.: Assembly and signaling of adhesion complexes. Curr. Top. Dev. Biol., 68, 183-225 (2005).

19. Li, L., Bennett, S.A., and Wang, L.: Role of E-cadherin and other cell adhesion molecules in survival and differentiation of human pluripotent stem cells, Cell Adh. Migr., 6, 59-70 (2012).

20. Liu, N., Lu, M., Tian, X., and Han, Z.: Molecular mechanisms involved in self-renewal and 
pluripotency of embryonic stem cells, J. Cell Physiol., 211, 279-286 (2007).

21. Li, D., Zhou, J., Wang, L., Shin, M.E., Su, P., Lei, X., Kuang, H., Guo, W., Yang, H., Cheng, L., Tanaka, T.S., Leckband, D.E., Reynolds, A.B., Duan, E., and Wang, F.: Integrated biochemical and mechanical signals regulate multifaceted human embryonic stem cell functions, J. Cell Biol., 191, 631-644 (2010).

22. Uda, Y., Poh, Y.C., Chowdhury, F., Wu, D.C., Tanaka, T.S., Sato, M., and Wang, N.: Force via integrins but not E-cadherin decreases Oct3/4 expression in embryonic stem cells, Biochem. Biophys. Res. Commun., 415, 396-400 (2011).

23. Yoshigi, M., Hoffman, L.M., Jensen, C.C., Yost, H.J., and Beckerle, M.C.: Mechanical force mobilizes zyxin from focal adhesions to actin filaments and regulates cytoskeletal reinforcement, J. Cell Biol., 24, 209-215 (2005).

24. Sims, J.R., Karp, S., and Ingber, D.E.: Altering the cellular mechanical force balance results in integrated changes in cell, cytoskeletal and nuclear shape, J. Cell Sci., 103, 1215-1222 (1992).

25. Galbraith, C.G., Yamada, K.M., and Sheetz, M.P.: The relationship between force and focal complex development, J. Cell Biol., 159, 695-705 (2002).

26. Jin, S., Yao, H., Weber, .JL., Melkoumian, Z.K., and Ye, K.: A synthetic, xeno-free peptide surface for expansion and directed differentiation of human induced pluriopotent stem cells, Plos One, 7, 1-10 (2012).

27. Hirata, H., Tatsumi, H., and Sokabe, M.: Mechanical forces facilitate actin polymerization at focal adhesions in a zyxin-dependent manner, J. Cell Sci., 121, 2795-2804 (2008). 


\section{Figure legends}

FIG. 1. Maintenance of undifferentiated hiPSCs by passaging colony cells on the G1 and gelatin-coated surfaces. (A) Immunostainings of four of pluripotency markers, Oct3/4, Nanog, SSEA-4 and Tra1-60 in hiPSCs cultured on G1 and gelatin-coated surfaces after 10 passages. Scale bars represent $250 \mu \mathrm{m}$. (B) Conventional RT-PCR (B1) and quantitative RT-PCR (B2) analyses for three markers of undifferentiated stem cells (Oct3/4, Nanog, Sox2) and early ectoderm (SOX1, NEUROD1), mesoderm (MESP1, T gene, EOMES), and endoderm (GATA4, GATA6, SOX17) differentiation markers. In quantitative RT-PCR analysis, closed and open bars show G1 and gelatin-coated surfaces, respectively. The data were obtained from three independent experiments. Statistical significance was according to Student's $t$-test $(* p<0.01)$.

FIG. 2. Secreted fibronectin from feeder cells co-cultured with hiPSCs on G1 and gelatin-coated surfaces for 5 days. (A) Immunostaining of fibronectin (green, A1 and A3) derived from feeder cells in central region of hiPSC colonies. Cell nuclei were stained with DAPI (blue, A2 and A4). Scale bars show $50 \mu \mathrm{m}$. (B) Conventional RT-PCR (upper panel) and quantitative RT-PCR (lower panel) analyses of fibronectin expression of feeder cells co-cultured with hiPSCs on G1 and gelatin-coated surfaces. In quantitative RT-PCR analysis, the data were obtained from three independent experiments, and analyzed using the Student's $t$-test. There are no statistical significances among the data sets.

FIG. 3. Immunostaining of paxillin (green) in hiPSC colonies cultured on G1 and gelatin-coated surfaces for 5 days. F-actin and cell nuclei were stained with rhodamine phalloidin (red) and DAPI (blue). Scale bars show $50 \mu \mathrm{m}$. Panels A-D show the three-dimensional images in of hiPSCs. Panels A2-D2 show merged enlargements of demarcated box areas in A-D, respectively. Panels A3-D3 show the side (cross-section) views in A2-D2, respectively. Feeder cells are indicated by the asterisk.

FIG. 4. Western blot analysis of paxillin, Rac1, and E-cadherin of hiPSCs cultured on G1 and 
gelatin-coated surfaces for 5 days.

FIG. 5. Immunostaining of zyxin (green) in hiPSC colonies cultured on G1 and gelatin-coated surfaces for 5 days. Cell nuclei were stained with DAPI (blue). Scale bars show $50 \mu \mathrm{m}$. Panels A and B show merged images of A1 and A2, and B1 and B2, respectively. Panels C and $\mathrm{D}$ show merged images of $\mathrm{C} 1$ and $\mathrm{C} 2$, and $\mathrm{D} 1$ and $\mathrm{D} 2$, respectively. 
A

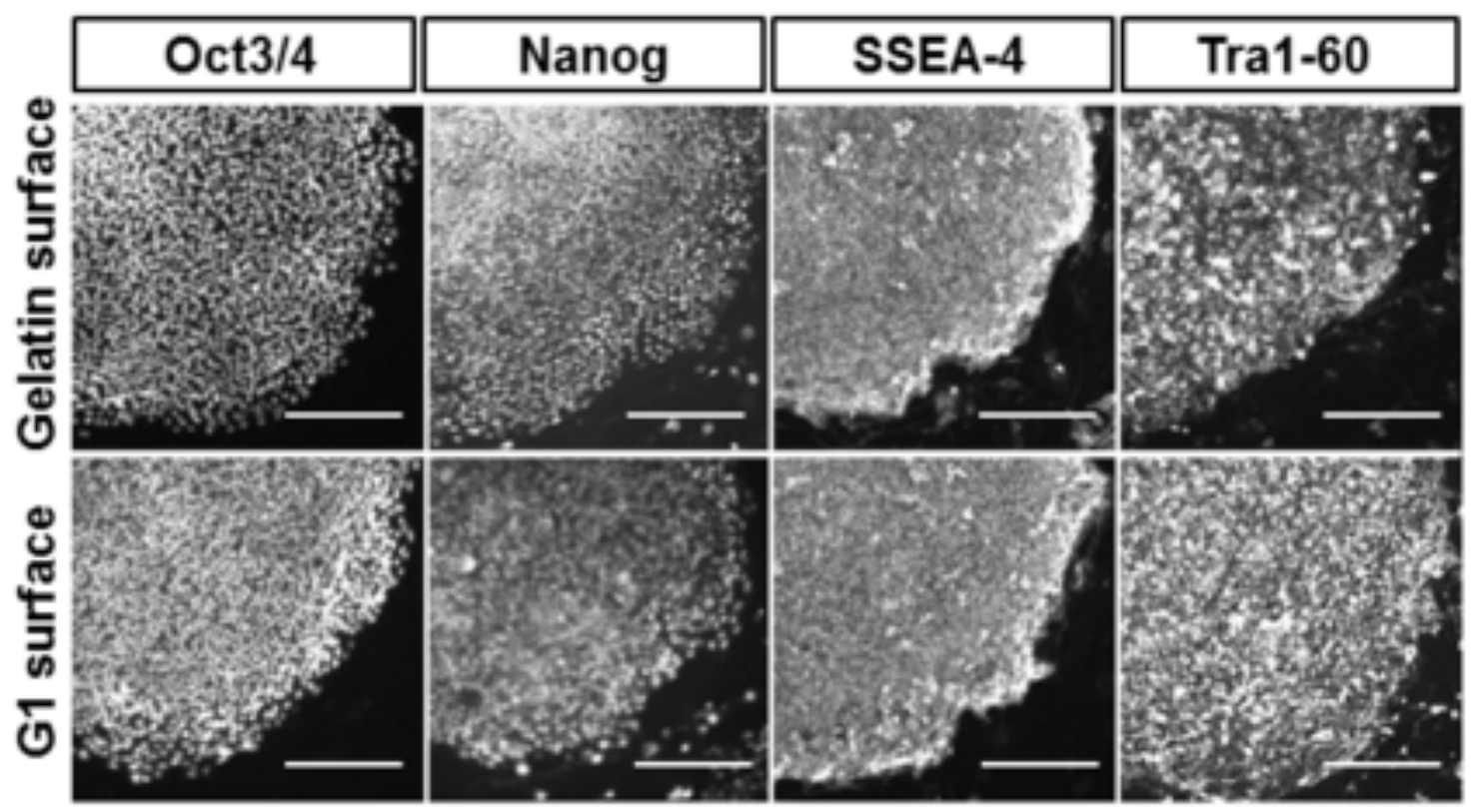

B
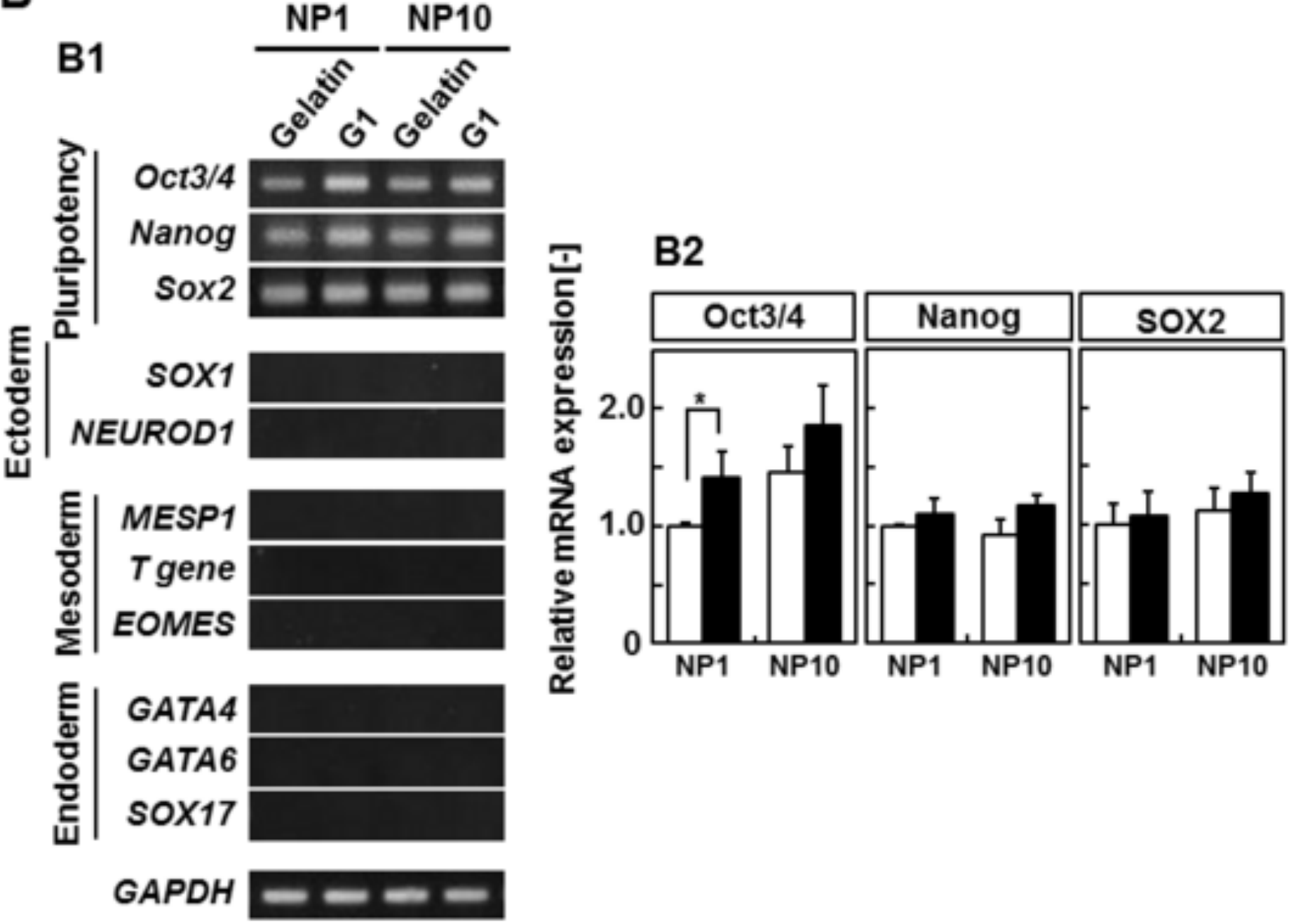
A

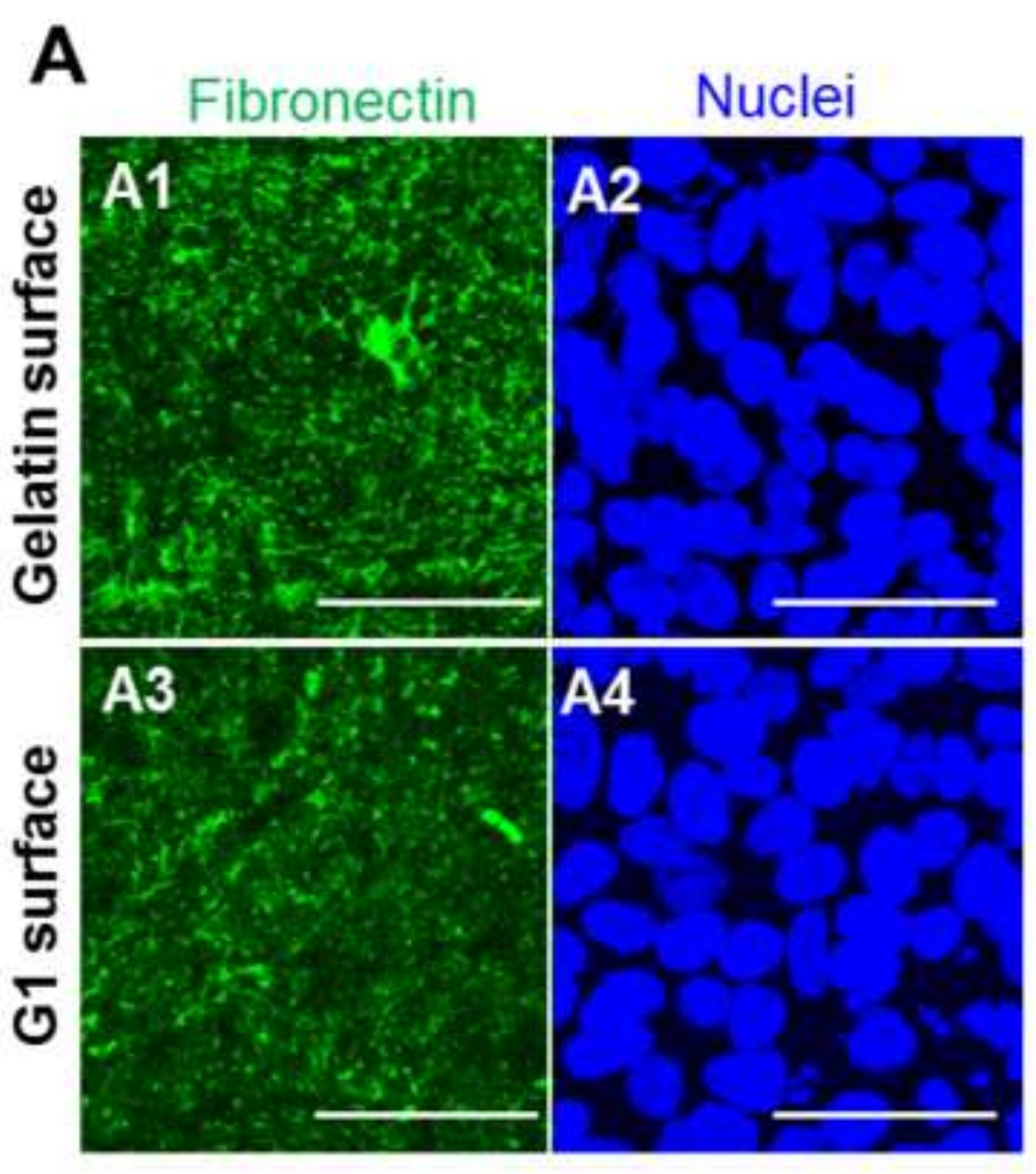

Fibronectin
B
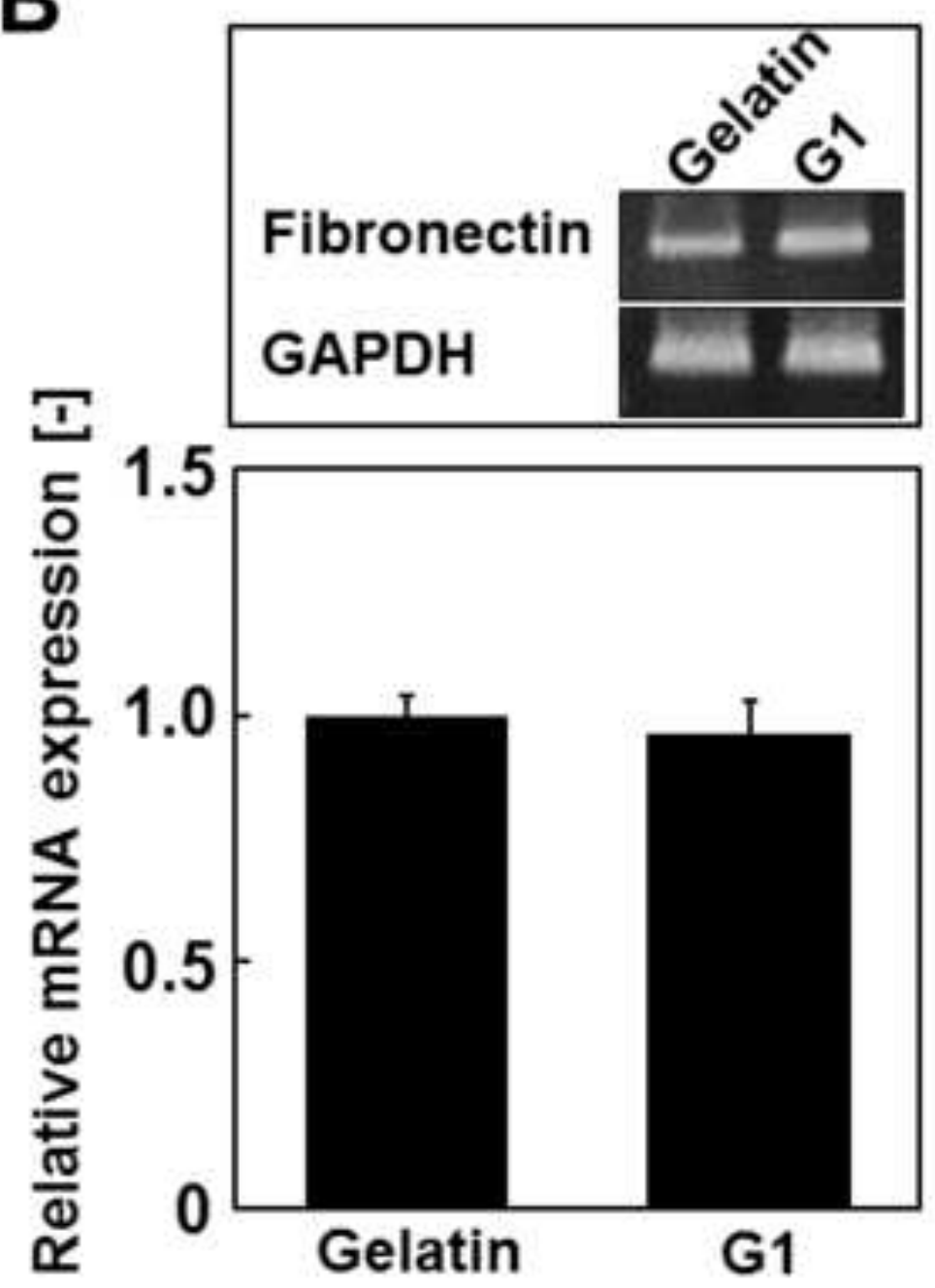

电 


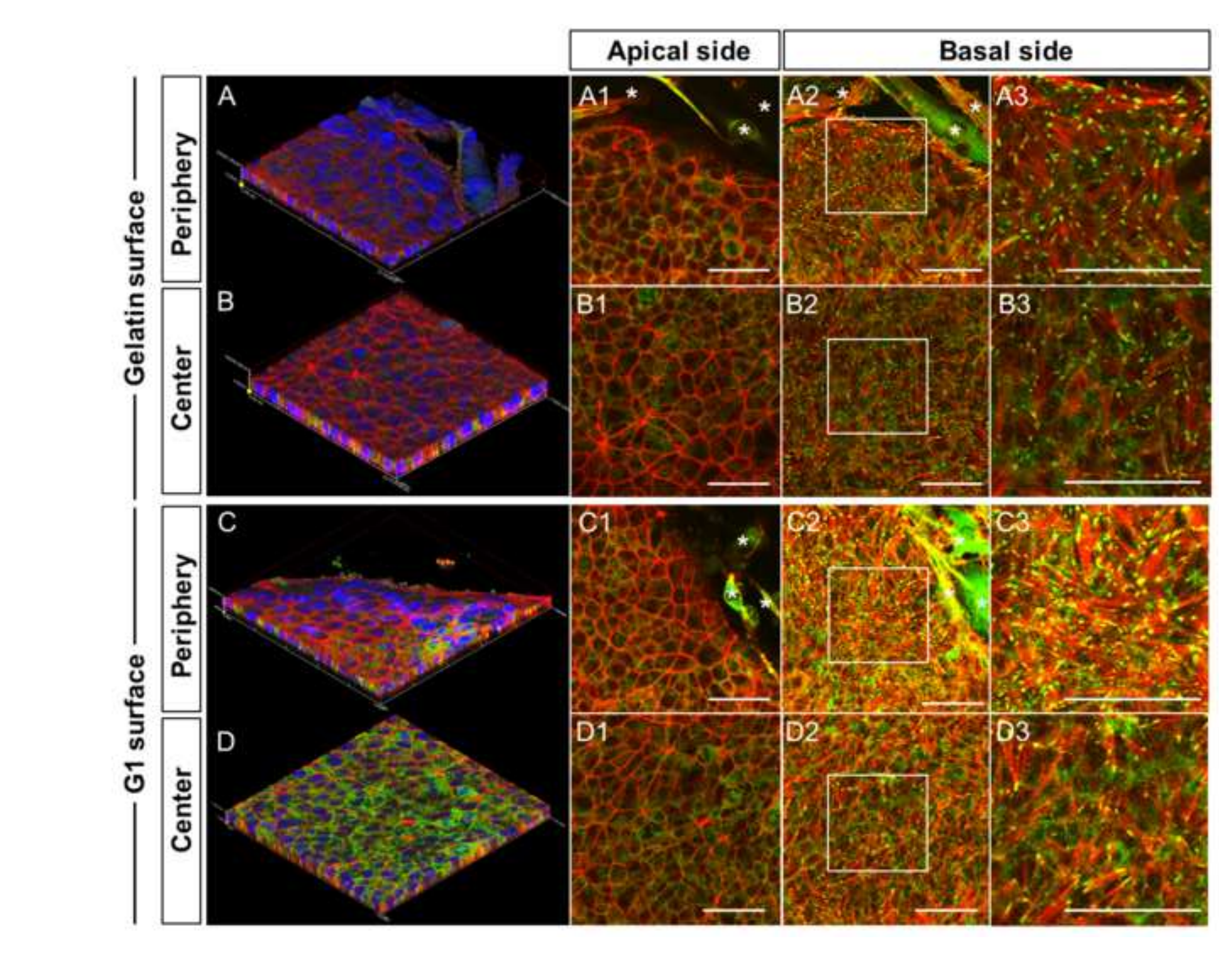
.

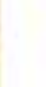

.

.

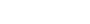




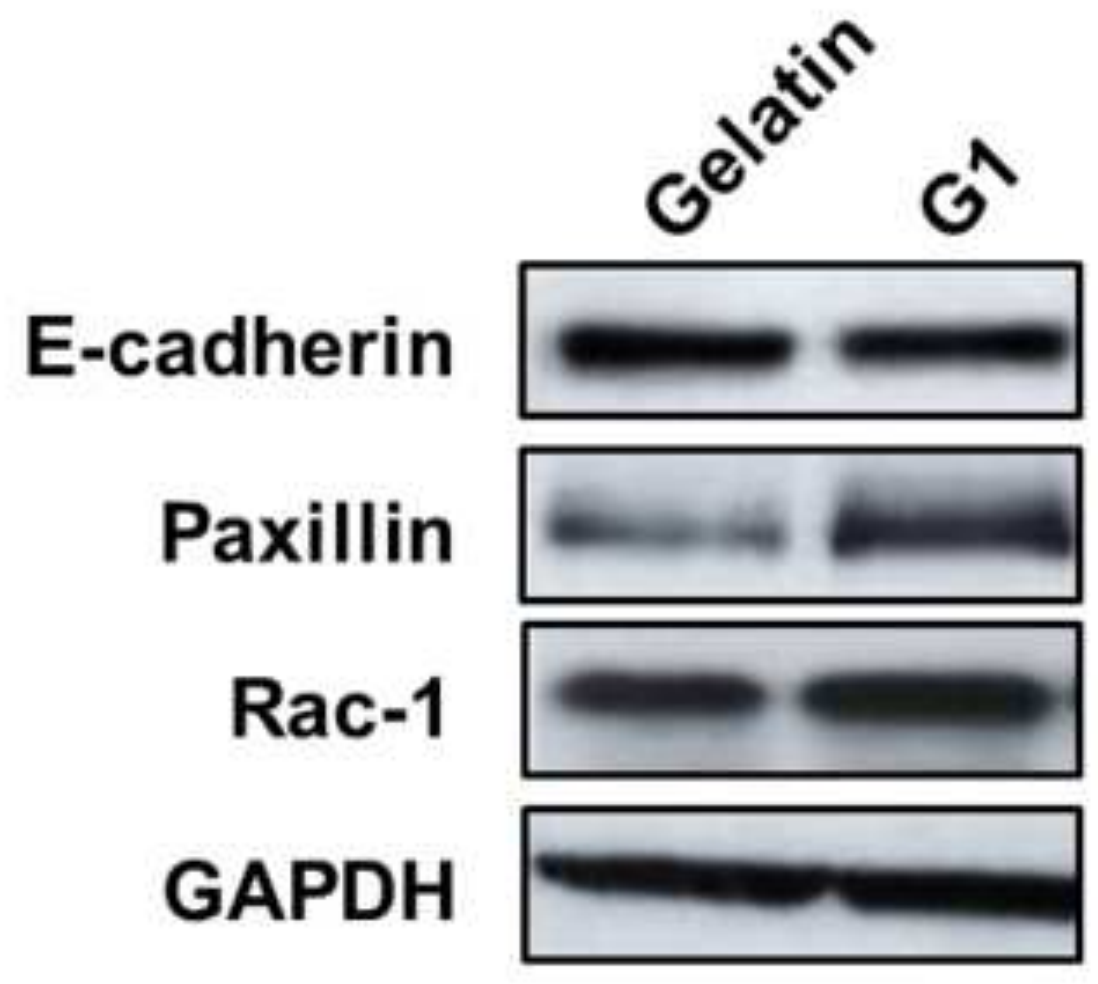

Figure 4

E-cadherin

Paxillin

Rac-1

GAPDH

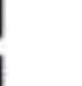

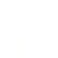

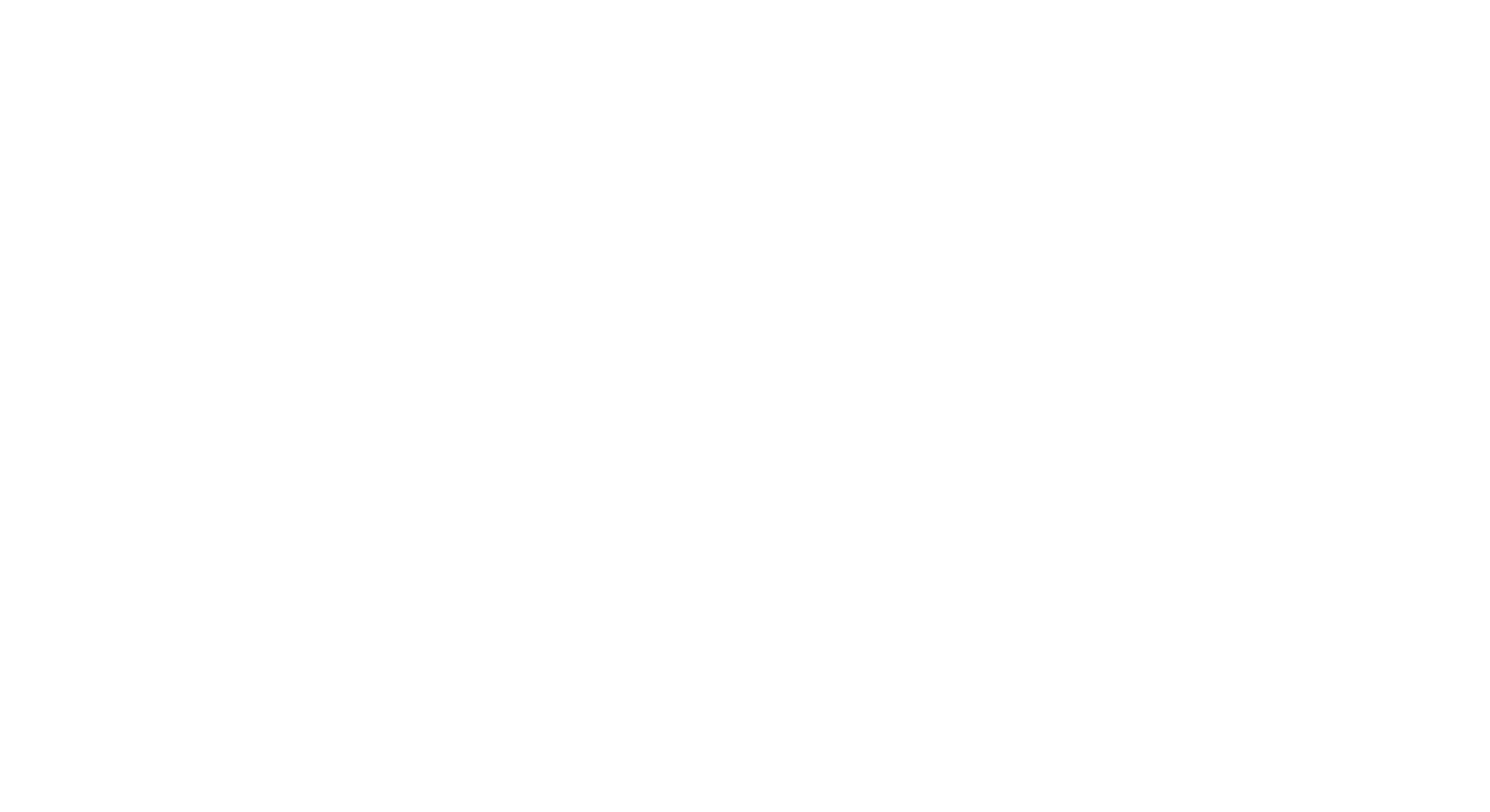
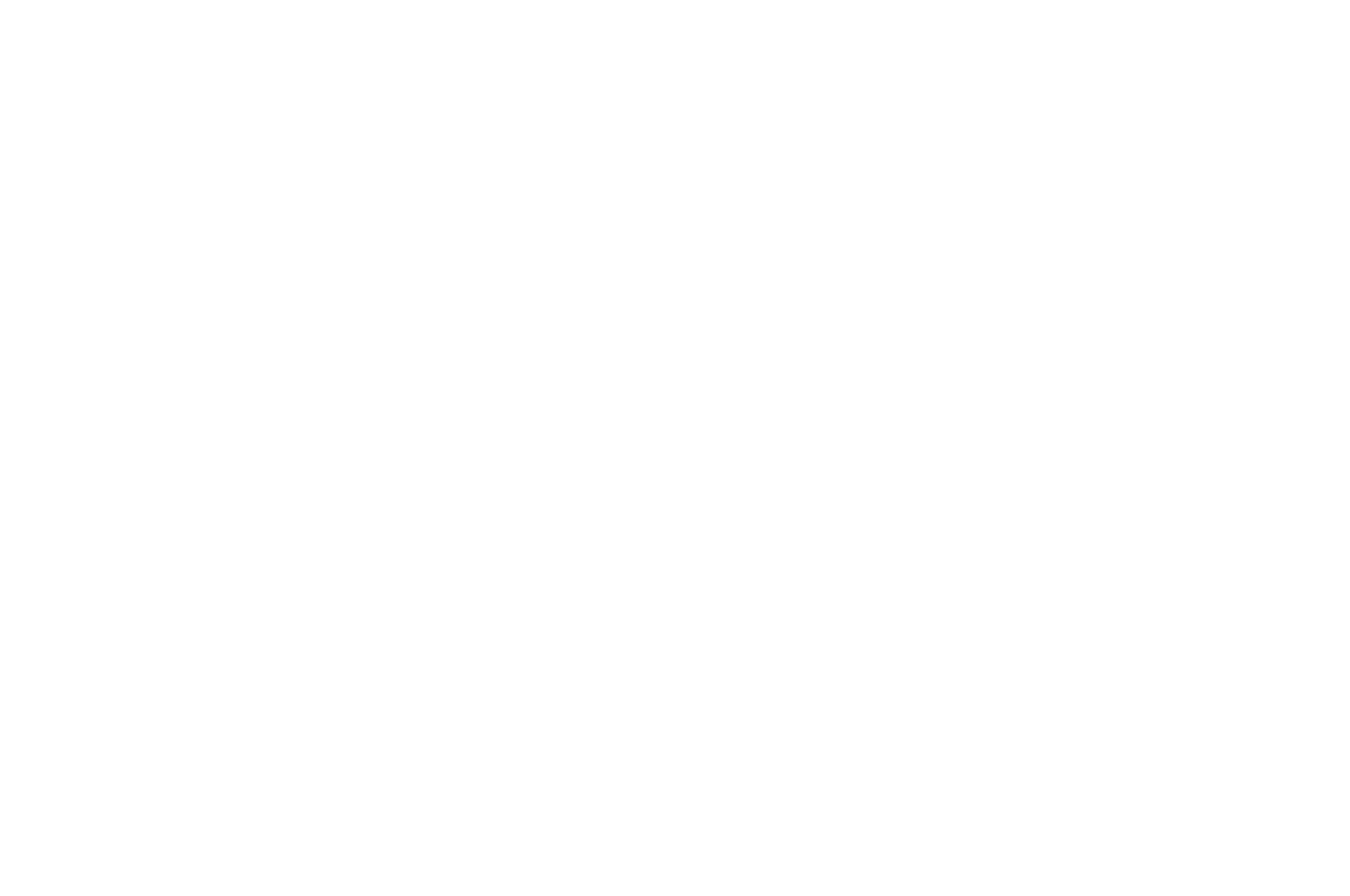


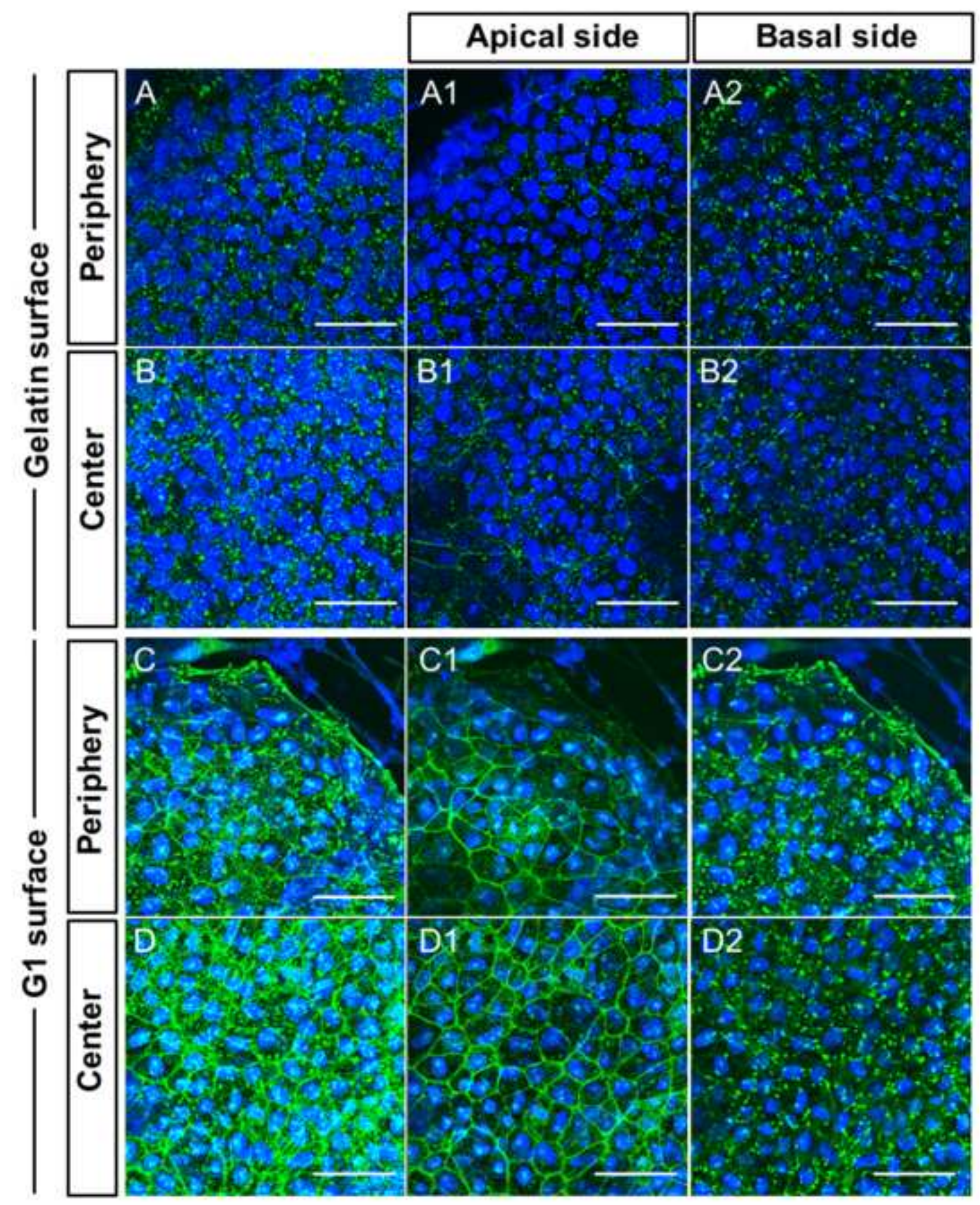

